\title{
Single-catheter approach for ablation of the slow pathway in a patient with type IV Ehlers-Danlos syndrome and AV nodal reentrant tachycardia using a magnetic navigation system
}

\author{
Tamas SZILI-TOROK, Emile JESSURUN, Luc J. JORDAENS \\ Department of Clinical Electrophysiology, Erasmus Medical Centre, Rotterdam, The Netherlands.
}

\begin{abstract}
Patients with Ehlers-Danlos syndrome type IV have thin-walled, friable arteries and veins. Invasive procedures carry a significantly increased risk for perforation of blood vessels. The aim of this case report is to demonstrate the feasibility and potential benefits of using a stereotactic magnetic navigation system (MNS) for mapping and ablation under these very special circumstances. A 45-yearold woman is presented with daily episodes of typical atrio-ventricular nodal re-entry tachycardias (AVNRT) and known Ehlers-Danlos syndrome type IV. Transcatheter ablation procedure of the AVNRT was undertaken using the MNS, with a non-traumatic single floppy catheter and the capability of advanced navigation.
\end{abstract}

Keywords: magnetic navigation - ablation - AVNRT.

\section{Introduction}

Patients with Ehlers-Danlos syndrome type IV have thin-walled, friable arteries and veins and are prone even to spontaneous arterial and venous ruptures ${ }^{1-3}$. Invasive procedures carry a significantly increased risk for perforation of blood vessels ${ }^{2}$. Especially in patients with non-life-threatening disorders this increased risk is not tolerable. We present a patient with very frequent, daily episodes of sustained AV nodal reentry tachycardias. Whereas medical treatment is only palliative, radiofrequency (RF) catheter ablation is curative in the majority of patients. Certainly, in the above mentioned patient population the advantage of curative treatment is counterbalanced by the significant risk of the endovascular approach. The recently developed magnetic navigation system (MNS) facilitates more precise control of ablation catheters in the treatment of complex arrhythmias ${ }^{4-9}$. More importantly, this catheter is an extremely floppy device, where perforation is virtually impossible. Recent data support the hypothesis that these systems may offer advantages to classic catheter ablation procedures. The aim of this case report is to demonstrate the feasibility and poten-

Address for correspondence: Tamas Szili-Torok, MD, PhD, Thoraxcentre, Department of Clinical Cardiac Electrophysiology, Erasmus MC, Rotterdam, The Netherlands. Dr Molewaterplein 40, kamer Ba 577, Postbus 2040, 3000 CA, Rotterdam, The Netherlands. E-mail: t.szilitorok@erasmusmc.nl

Received 13 November 2007; revision accepted for publication 20 March 2008 . tial benefits of using a stereotactic MNS for mapping and ablation under these very special circumstances.

\section{Case presentation}

This 45-year-old woman had daily episodes of sustained narrow complex QRS tachycardias. Her 12-lead ECG recorded during the tachycardia was very suggestive of AV nodal re-entrant tachycardia, showing a regular narrow complex tachycardia, with pseudo $r$ waves in lead V1 and pseudo q waves in leads II, III and aVF (figure 1A). Clinically, it required several times intravenous administration of adenosine because of the incessant nature of the arrhythmia. The patient was formerly diagnosed with Ehlers-Danlos syndrome (vascular type, type IV) because of previous vascular complications related to pregnancy. Later she had spontaneous development of bowel perforations and fistulas. The patient was informed about the availability of the novel MNS at our centre and we undertook a single, floppy catheter ablation approach for elimination of her slow pathway. The procedure was performed under deep sedation.

\section{MNS SYSTEM AND ELECTROPHYSIOLOGY STUDY}

Through the femoral vein a magnetic navigation quadripolar catheter (Celsius-RMT $4 \mathrm{~mm}$, BiosenseWebster, Inc, Diamond Bar, CA, USA) was introduced and advanced into the right atrium using the automatic 
A

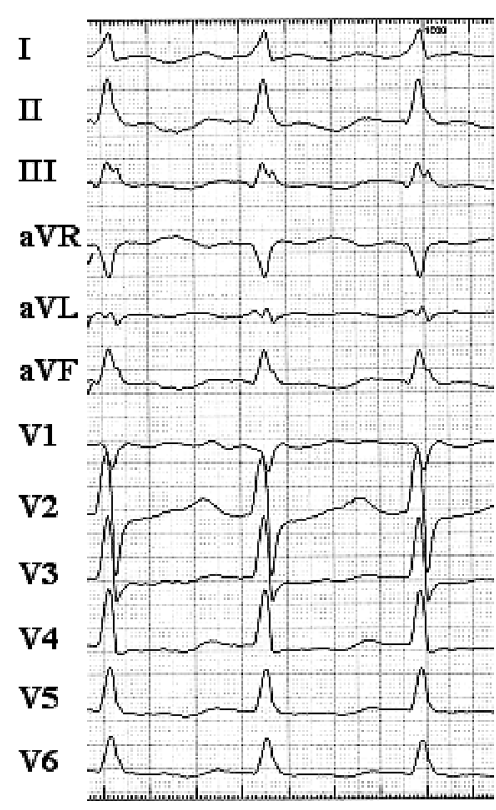

B

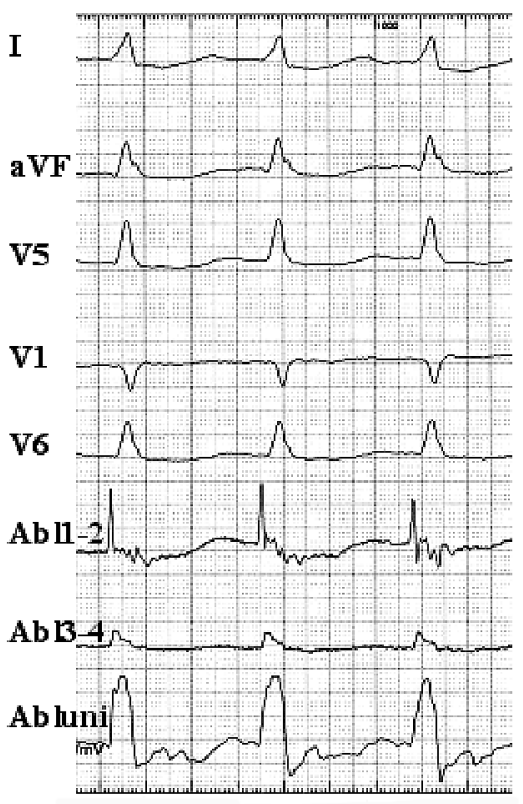

C

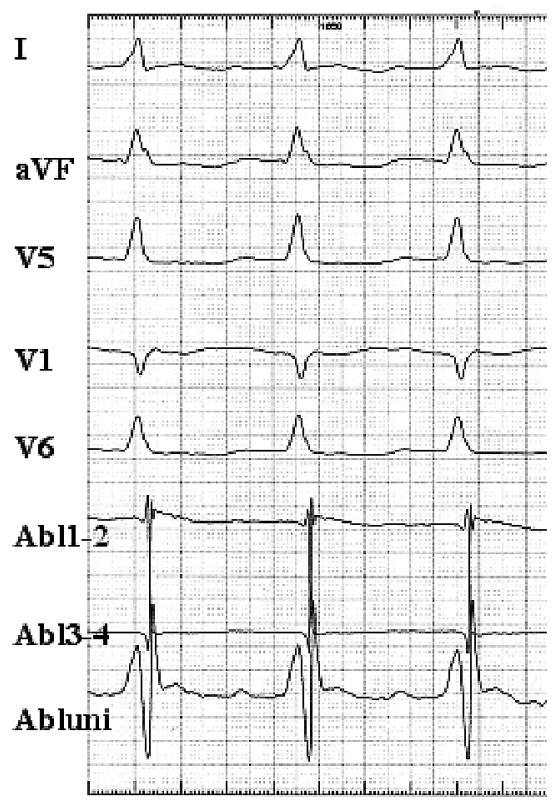

Fig. 1. - A: 12-lead ECG of the patient showing a narrow QRS complex tachycardia suggestive of AVNRT. B and C: Electrograms obtained from the proximal and distal CS during tachycardia showing central retrograde conduction with a very short VA time. All tracings are recorded with a paper speed of $50 \mathrm{~mm} / \mathrm{s}$.

catheter advancer system (Cardiodrive ${ }^{\mathrm{TM}}$, Stereotaxis, Stereotaxis Inc, St Louis, MO, US) placed in the patient's thigh. The catheter was manoeuvred using the MNS (Niobe Stereotaxis MNS, Stereotaxis Inc.). The Niobe system consists of two large permanent magnets, situated on either side of the patient and controlled via a computer workstation (Navigant, Stereotaxis Inc.) to allow for changes in the orientation of a stable magnet field within the chest of the patient. The ablation catheter has 3 magnets in the distal shaft that align themselves with the field produced by the external magnets, allowing for effective catheter orientation. Remote catheter advancement and retraction was performed with the catheter advancer system. After the magnets were brought in next to the patient, remote control of all of the components of the system was performed by the physicians from the control room.

During the electrophysiology study we utilized the potential advantages of the magnetic navigation system in accurate navigation, the possibility of storage of magnetic vectors to certain locations and the use of floppy non-traumatic catheters. Initially the catheter was introduced to a right atrium. Using vectors towards the lateral wall a right atrial lateral position was achieved. At this position the atrial signal amplitude was large, and there was a low stimulation threshold. Then the His position was reached. The catheter was placed into this His region providing ventricular capture on the distal electrode pairs with wide-paced QRS duration, while on the proximal pole a clear atrial signal was still visible. After this, the coronary sinus was cannulated using the navigation system. All magnetic vectors of the above mentioned anatomical locations were stored in the system (figure 2). This allowed quick re-navigation of the catheter to a selected spot using the system's target navigation option. After registration of the important anatomical locations, ventricular programmed stimulation was performed which clearly showed decremental conduction. During pacing from the right atrium there was an AQRS jump, reproducibly seen with atrial echos. Incremental pacing from this site easily induced the tachycardia. During tachycardia the catheter was again re-navigated to the His position, into the CS proximal and distal part retrospectively (figure 2). The VA time was less than $90 \mathrm{~ms}$ at all of these locations. The proximal CS A signal was earlier than the distal CS A (figure $1 \mathrm{~B}$ and $1 \mathrm{C})$. Finally, during ventricular pacing adenosine was administered (18 $\mathrm{mg}$ intravenously) which resulted in VA dissociation. Based on the findings of the EP study, AVNRT was diagnosed and slow pathway ablation was undertaken. The fluoroscopy image was set to LAO 30 degrees, and the navigation started at the His position. The magnetic vector was changed in the same plane inferior from the His, providing safe distance from the His bundle. Movement of the catheter was continuously monitored and recorded. When an optimal balance of a small fractionated A and a large $V$ signal was seen, RF energy was applied starting at a low energy level. At the first ablation spot accelerated junctional rhythm was observed. After this single application the tachycardia was no longer inducible, not even in the presence of isoproterenol. The A-QRS jump disappeared and there were no more 

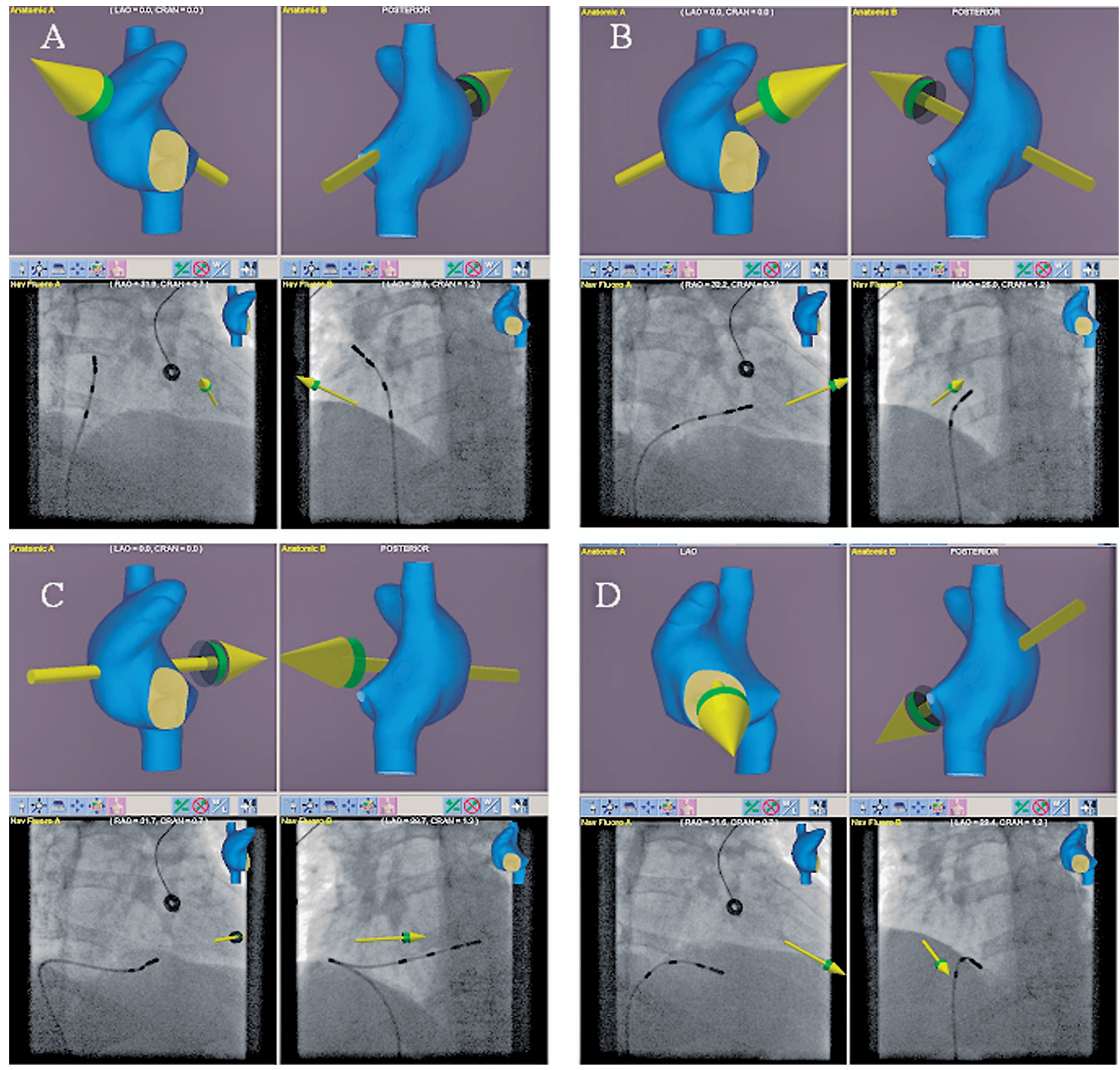

Fig. 2. - Catheter positions and magnetic vectors during mapping and during RF ablation of AVNRT. The green arrows represent the desired direction of the catheter positions, while yellow vectors show the actually applied magnetic vector. All panels contain LAO $\left(30^{\circ}\right)$ and posterior views of the right atrial model. Lower panels demonstrate LAO $\left(30^{\circ}\right)$ and RAO $\left(30^{\circ}\right)$ views of fluoroscopy images. Panel A: Catheter position in the high right atrium as achieved by the magnetic vector direction displayed on the virtual anatomy. Panel B: His position. Panel C: distal coronary sinus. Panel D: catheter position in slow pathway region, at the ablation site.

echo beats. Because of her connective tissue disorder the patient was kept in the hospital for three additional days. There were no complications related to the procedure. There were no tachycardias on telemetry. She had frequent atrial premature beats without the induction of the tachycardia. At 6-month follow-up the patient was completely free from tachycardias.

\section{Comments}

Most of the recent publications related to the stereotaxis magnetic navigation system deal with fea- sibility issues. Indeed, multiple papers show that this novel system can be used to treat most of the arrhyth$\operatorname{mias}^{5-7,9}$. Although the feasibility is not questionable anymore, the new technology is only accepted fully, if it provides clear advantages. There are theoretical advantages of using magnetic navigation. However, until recently there is no clear evidence supporting its use, except for the reduced radiation exposure to the treating physician. There are additional factors, which can provide further benefit. One of the potential advantages of the system is the utilization of a nontraumatic catheter. This catheter is very floppy, therefore it reduces the risk of perforation. Our case is 
indeed an extreme example of this, since vascular type (type IV) Ehlers-Danlos syndrome is frequently associated with complications related to vessel and/or internal organ perforation. However, it might have an impact on the complication rate of standard ablation procedures if enough patients are studied. Another suggestion by this case report is actually to use the superior navigation capabilities to reduce the cost of the procedure. Although this was definitely not the aim in this patient, one should note that this single-catheter approach might decrease the overall procedural cost. The latter issue can be addressed with a feasibility study, where certainly non-inferiority in success rate and complication rate should be demonstrated.

\section{References}

1. Gilchrist ER, Duflou JA. Incidental myocardial infarction in Ehlers-Danlos syndrome type IV? J Forensic Sci 2005; 50: 461-4.

2. Brearley S, Fowler J, Hamer JD. Two vascular complications of the Ehlers-Danlos syndrome. Eur J Vasc Surg 1993; 7: 210-3.

3. Evans RH, Fraser AG. Spontaneous coronary artery rupture and cardiac tamponade in Ehlers-Danlos syndrome type IV. Int J Cardiol 1996; 54: 283-6.
4. Davis DR, Tang AS, Birnie DH, Gollob MH. Successful ablation of a concealed parahisian accessory pathway using a remote magnetic navigation system following failure by conventional methods. J Interv Card Electrophysiol 2006; 16: 149-51.

5. Ernst S, Ouyang F, Linder C, Hertting K, Stahl F, Chun J, Hachiya H, Bänsch D, Antz M, Kuck KH. Initial experience with remote catheter ablation using a novel magnetic navigation system: magnetic remote catheter ablation. Circulation 2004; 109: 1472-5.

6. Pappone C, Vicedomini G, Manguso F, Gugliotta F, Mazzone P, Gulletta S, Sora N, Sala S, Marzi A, Augello G, Livolsi L, Santagostino A, Santinelli V. Robotic magnetic navigation for atrial fibrillation ablation. $\mathrm{J} \mathrm{Am} \mathrm{Coll} \mathrm{Cardiol}$ 2006; 47: 1390-400.

7. Pflaumer A, Hessling G, Luik A, Wu J, Zrenner B. Remote magnetic catheter mapping and ablation of permanent junctional reciprocating tachycardia in a seven-year-old child. J Cardiovasc Electrophysiol 2007; 18: 882-5.

8. Thornton AS, Jordaens L. A left-sided accessory pathway revisited with remote retrograde magnetic navigation. Pacing Clin Electrophysiol 2007; 30: 573-6.

9. Thornton AS, Rivero-Ayerza M, Knops P, Jordaens LJ Magnetic navigation in left-sided AV reentrant tachycardias: preliminary results of a retrograde approach. $J$ Cardiovasc Electrophysiol 2007; 18: 467-72. 